\title{
COVID-19 Rapid Antigen Test: Role in Screening Prior to Gastrointestinal Endoscopy
}

\author{
Ashok Dalal, Ujjwal Sonika, Manish Kumar, Roshan George, Ajay Kumar, Siddharth Srivastava, Sanjeev Sachdeva and Barjesh \\ Chander Sharma
}

Department of Gastroenterology, Govind Ballabh Pant Institute of Postgraduate Medical Education and Research (GIPMER), New Delhi, India

Background/Aims: The severe acute respiratory syndrome coronavirus 2 pandemic has affected the gastrointestinal (GI) endoscopy units globally owing to the risk of transmission. We present our data on the use of rapid antigen test (RAT) as a screening tool prior to endoscopy to prevent the transmission of coronavirus disease (COVID-19).

Methods: This study was a retrospective analysis of patients who underwent any GI endoscopic procedure from July 2020 to October 2020 at a tertiary referral center in New Delhi, India. All patients underwent screening for COVID-19 using RAT, and endoscopy was performed only when the RAT was negative. The data are presented as numbers and percentages.

Results: A total of 3,002 endoscopic procedures were performed during the study period. Only one endoscopic procedure was performed in a COVID-19 positive patient. A total of 53 healthcare workers were involved in conducting these procedures. Only 2 healthcare workers (3.8\%) were diagnosed COVID-19 positive, presumably due to community-acquired infection, during this period.

Conclusions: The COVID-19 RAT is easily usable as a simple screening tool prior to GI endoscopy during the COVID-19 pandemic. Clin Endosc 2021;54:522-525

Key Words: Cholangiopancreatography, endoscopic retrograde; COVID-19; Endoscopy; SARS-COV-2 antigen testing

\section{INTRODUCTION}

Coronavirus disease (COVID-19) is caused by the novel coronavirus, severe acute respiratory syndrome coronavirus 2 (SARS-CoV-2), and is primarily transmitted through respiratory droplets and aerosols. It was declared as a global pandemic by the World Health Organization (WHO) in March $2020{ }^{1}$ Healthcare workers in gastrointestinal (GI) endoscopy units

\footnotetext{
Received: November 14, 2020 Revised: December 12, 2020

Accepted: December 23, 2020

Correspondence: Ujjwal Sonika

Department of Gastroenterology, Govind Ballabh Pant Institute of Postgraduate Medical Education and Research (GIPMER), Academic Block, 1, JLN Marg, New Delhi 110002, India

Tel: +91-11-23232013, Fax: +91-11-23239442, E-mail: usonika@gmail.com ORCID: https://orcid.org/0000-0003-0949-2382
}

(c) This is an Open Access article distributed under the terms of the Creative Commons Attribution Non-Commercial License (http://creativecommons.org/ licenses/by-nc/3.0) which permits unrestricted non-commercial use, distribution, and reproduction in any medium, provided the original work is properly cited. are at an increased risk of transmission, as endoscopic procedures generate large amounts of aerosols. ${ }^{2}$ Moreover, endoscopists are positioned very close to the patient's mouth and nose throughout the procedure, which can last from a few minutes to an hour, depending on the complexity of the procedure. ${ }^{3}$ Hence, it is of utmost importance to prevent the transmission of COVID-19 infection from the patient to the healthcare workers or vice-versa.

The current guidelines recommend testing for COVID-19 within 24 to 72 hours prior to the endoscopic procedures. ${ }^{4}$ The reverse transcription polymerase chain reaction (RT-PCR) is recommended by most of the guidelines for screening, as it has the highest sensitivity and specificity. However, it is expensive and can take up to $24 \mathrm{hr}$ until the test results of the RTPCR assay are available. Rapid antigen tests (RATs) are being increasingly used for COVID-19 screening, because they are low-cost, and the test results are available almost instantaneously. The RATs have high specificity (99.3\% to $100 \%)$; however, the sensitivity varies from $50.6 \%$ to $84 \%$ depending 
on the viral load. ${ }^{5,6}$

RAT testing as a screening strategy prior to endoscopic procedures for the prevention of COVID-19 transmission has previously not been evaluated. In this study, we evaluated the role of COVID-19 RAT testing prior to endoscopic procedures for the prevention of SARS-CoV-2 transmission in our endoscopy unit.

\section{MATERIALS AND METHODS}

This retrospective study was conducted at the Department of Gastroenterology, GB Pant Institute of Post Graduate Medical Education and Research, New Delhi. Ethical clearance was waived-off by the institutional ethics committee in view of the retrospective nature of the study. All patients visiting the outpatient department (OPD) underwent COVID-19 screening using Rapid Antigen Tests (Standard Q Rapid Test; SD BIOSENSOR, Suwon, Korea). Patients who were RAT negative were allowed into the OPD, and all the positive patients were referred to the dedicated hospital for COVID-19 patients. The patients underwent endoscopic procedures at our endoscopy unit as advised by the treating clinician. Patients whose endoscopic procedures were performed after more than 1 week of the initial screening RAT, underwent a repeat RAT, and the procedure was performed if the second RAT was negative. Emergency endoscopic procedures were planned for COVID-19 positive patients at a separate dedicated area of the hospital. Elective procedures for COVID-19 positive patients were deferred.

During the endoscopic procedures, all staff involved in the endoscopy wore a disposable waterproof surgical gown, gloves, shoe-cover, face-shield, and an N-95 mask. Complete personal protection equipment (PPE) kits, including waterproof hazmat suit with head cover, N-95 mask, double gloves, and goggles, were used if the patient was COVID-19 positive.

The healthcare workers were tested for COVID-19 when symptomatic or if there was an exposure to confirmed COVID-19 patients either at healthcare facilities or in the community. No routine screening tests for COVID-19 were performed for the healthcare workers. COVID-19 negative patients were not routinely tested for COVID-19 post-procedure.

The data were collected from the endoscopy unit registers from July 2020 to October 2020. The data are presented as numbers and percentages.

\section{RESULTS}

A total of 3,002 endoscopic procedures were performed during the period from July 1, 2020, till October 31, 2020, in our GI endoscopy unit. They included a total of 2052, 444, 424 , and 82 procedures of upper GI endoscopy, lower GI endoscopy, endoscopic retrograde cholangiopancreatography (ERCP), and endoscopic ultrasound (EUS), respectively (Table 1). As expected, there was a marked reduction in the number of procedures performed this year compared those performed last year. A total of 4,027, 969, 951, and 221 underwent upper GI endoscopy, lower GI endoscopy, ERCP, and EUS respectively, from July 2019 to October 2019.

Only one endoscopic procedure was performed for a COVID-19 positive male patient at a designated COVID-19 area with complete PPE protection, which included a waterproof Hazmat suit with headcover, N-95 mask, double gloves, and goggles. The patient presented with hematemesis, and endoscopic examination revealed extensive gastric erosion. No endotherapeutic procedure was performed. All other endoscopic procedures were performed for patients who were COVID-19 negative per the results of the RAT.

All endoscopic procedures were performed by six consultants and nine fellows undergoing training. A total of 38 support staff (nurses and workers) assisted with the procedures. Thus, a total of 53 healthcare workers were involved in the functioning of endoscopy units. Two (3.8\%) healthcare workers (one nurse and one worker) tested positive for COVID-19 during this period; however, both had a history of exposure to a confirmed COVID-19 patient in the community. No

Table 1. List of Endoscopic Procedures Done Month-Wise

\begin{tabular}{lccccc}
\hline Type of procedure & July & August & September & October & Total \\
\hline Upper GI endoscopy & 395 & 450 & 631 & 576 & 2,052 \\
Lower GI endoscopy & 90 & 86 & 142 & 126 & 444 \\
ERCP & 87 & 72 & 120 & 145 & 424 \\
Endoscopic ultrasound & 2 & 2 & 36 & 42 & 82 \\
\hline
\end{tabular}

ERCP, endoscopic retrograde cholangiopancreatography; GI, gastrointestinal. 
other healthcare worker had a high-risk exposure to either of these two COVID-19 positive workers and thus, was not quarantined or tested for COVID-19. High-risk exposure is defined as having face-to-face contact (without mask) with a COVID-19 case within $1 \mathrm{~m}$ for more than 15 minutes. ${ }^{7}$ Both workers were home quarantined and reported back to duty after the quarantine period of 17 days. None of the other staff members had symptoms of COVID-19 during the study period. Despite being asymptomatic, eight other healthcare workers were tested for COVID-19 for suspected exposure in the community, and all of them tested negative.

\section{DISCUSSION}

Our study shows that the RAT for COVID-19 is an easy, economical, and convenient screening tool for patients undergoing GI endoscopic procedures. During the study period, the COVID-19 pandemic was raging in New Delhi, with the number of confirmed COVID-19 cases being 48,238, 39,150, 104,967, and 106,991 in July, August, September, and October, 2020, respectively, according to the data provided by the Delhi government "on their twitter handle @CMOdelhi". We reported only two cases of confirmed COVID-19 among the healthcare workers, which were probably related to community transmission. Endoscopic procedures are associated with a considerable amount of aerosol generation, and the endoscopist remains in close vicinity of the patient throughout the procedure. However, no endoscopist developed symptoms during the study duration. Some healthcare workers who were tested despite being asymptomatic were found to be negative.

The tests used to diagnose COVID-19 are nucleic acid-based tests such as RT-PCR and antigen-based tests such as RAT. Serological tests that detect antibodies to SARS-CoV-2 antigens such as spike proteins do not identify the active infection and thus are not used for the diagnosis of COVID-19. However, they are used to conduct serosurveys to determine the seroprevalence in population-based studies.

The advantage of RAT is that it can be used in emergency situations in the endoscopy unit, as no special training is required to perform the tests, and results are obtained almost instantaneously. It is also more economical and less labor-intensive, which is a significant advantage in resource-limited settings. It can provide a template or road map for reopening of endoscopic units throughout the world as the COVID-19 pandemic seems unlikely to subside in the near future.

The strength of our study is that all patients were screened universally prior to the procedure. A wide variety of procedures were performed in large numbers, including diagnostic and therapeutic upper GI procedures, colonoscopies, ERCP, and endoscopic ultrasonographies. Another strength of this study is that the trainees also performed a substantial number of these procedures. Our results will enhance the confidence of GI endoscopy training centers across the world to continue training the GI fellows.

The limitations of our study are that no routine screening for COVID-19 was performed for healthcare workers and patients after the procedure. This underestimates the transmission of COVID-19 in our endoscopy unit; nevertheless, it also highlights that the clinically significant symptomatic infections are few and can be prevented by universal screening using RAT prior to GI endoscopic procedures.

We conclude that the COVID-19 RAT is a simple, economical, and convenient screening tool prior to GI endoscopy procedures during the COVID-19 pandemic.

Conflicts of Interest

The authors have no potential conflicts of interest.

Funding

None.

Author Contributions

Conceptualization: Ashok Dalal, Ujjwal Sonika, Ajay Kumar, Sanjeev Sachdeva

Data curation: Manish Kumar, Roshan George

Methodology: US,AK

Writing-original draft: AD, US

Writing-review\&editing: Siddharth Srivastava, SSa, Barjesh Chander Sharma

$\begin{array}{ll}\text { ORCID } & \\ \text { Ashok Dalal: } & \text { https://orcid.org/0000-0001-7590-2226 } \\ \text { Ujjwal Sonika: } & \text { https://orcid.org/0000-0003-0949-2382 } \\ \text { Manish Kumar: } & \text { https://orcid.org/0000-0001-9994-2919 } \\ \text { Roshan George: } & \text { https://orcid.org/0000-0003-1496-9480 } \\ \text { Ajay Kumar: } & \text { https://orcid.org/0000-0002-5877-4610 } \\ \text { Siddharth Srivastava: } & \text { https://orcid.org/0000-0001-7550-0358 } \\ \text { Sanjeev Sachdeva: } & \text { https://orcid.org/0000-0002-5588-1111 } \\ \text { Barjesh Chander Sharma: } & \text { https://orcid.org/0000-0002-5878-2661 }\end{array}$

\section{REFERENCES}

1. Ludwig S, Zarbock A. Coronaviruses and SARS-CoV-2: a brief overview. Anesth Analg 2020;131:93-96.

2. Rana SS. Risk of COVID-19 transmission during gastrointestinal endoscopy. Journal of Digestive Endoscopy 2020;11:27-30.

3. Gralnek IM, Hassan C, Beilenhoff U, et al. ESGE and ESGENA position statement on gastrointestinal endoscopy and COVID-19: an update on guidance during the post-lockdown phase and selected results from a membership survey. Endoscopy 2020;52:891-898.

4. Sultan S, Siddique SM, Altayar O, et al. AGA institute rapid review and recommendations on the role of pre-procedure SARS-CoV-2 testing and endoscopy. Gastroenterology 2020;159:1935-1948.e5. 
5. Indian Council of Medical Research. Advisory on use of rapid antigen detection test for COVID-19 [Internet]. New Delhi: ICMR; c2020 [updated 2020 Jun 14]. Available from: https://www.icmr.gov.in/pdf/covid/ strategy/Advisory_for_rapid_antigen_test14062020.pdf.

6. Scohy A, Anantharajah A, Bodéus M, Kabamba-Mukadi B, Verroken A, Rodriguez-Villalobos H. Low performance of rapid antigen detection test as frontline testing for COVID-19 diagnosis. J Clin Virol
2020;129:104455

7. Ministry of Health \& Family Welfare. Advisory for managing health care workers working in COVID and non-COVID areas of the hospital [Internet]. New Delhi: MoHFW; c2020 [updated 2020 May 15]. Available from: https:/www.mohfw.gov.in/pdf/AdvisoryformanagingHealthcareworkersworkinginCOVIDandNonCOVIDareasofthehospital.pdf. 